\title{
Medicina do Estilo de Vida na educação médica: uma revisão narrativa
}

\author{
Lifestyle Medicine in medical education: a narrative review
}

Medicina de estilo de vida em la educación médica: una revisión narrativa

Edienny Augusta Viana Santos-Lobato ${ }^{1 *}$, Bruno Lopes dos Santos-Lobato ${ }^{1,2}$, Milena Coelho Fernandes Caldato ${ }^{1}$.

\section{RESUMO}

Objetivo: O objetivo desta revisão foi identificar evidências científicas relacionadas ao uso de estratégias de implementação de um programa em Medicina do Estilo de Vida nas Escolas Médicas. Revisão Bibliográfica: A Medicina do Estilo de Vida (MEV) é uma especialidade médica que enfatiza o aconselhamento médico sobre comportamentos que podem melhorar a saúde e qualidade de vida de pacientes. Os pilares desta metodologia incluem aconselhamento alimentar, aconselhamento em atividade física, mudança de comportamento, saúde do sono, cessação do tabagismo, uso responsável do álcool, bem-estar emocional e redução do estresse. Após a publicação de competências em MEV para educação médica em 2010, um número considerável de escolas médicas tem implementado avanços curriculares neste tema, que iniciam desde atividades de aprendizagem para seguimento longitudinal de pacientes sob à ótica da MEV, até a completa integração curricular, inserindo competências em MEV a todos os eixos curriculares já existentes. Considerações Finais: O desenvolvimento de um currículo em MEV é viável e a análise precoce destas estratégias mostra que os estudantes apresentam melhora na confiança quanto a realização de prescrições e orientações em mudanças de estilo de vida para pacientes com fatores de risco para doenças crônicas não transmissíveis.

Palavras-chave: Estilo de Vida, Educação Médica, Currículo

\begin{abstract}
Objective: The objective of this review was to identify scientific evidence related to the use of strategies to implement a Lifestyle Medicine program in medical schools. Literature Review: Lifestyle Medicine is a medical specialty that emphasizes medical advice on behaviors that can improve health and quality of life of patients. Pillars of this methodology include dietary counseling, physical activity counseling, behavior change, sleep health, smoking cessation, responsible alcohol use, emotional well-being and stress reduction. Following the publication of Lifestyle Medicine competences for medical education in 2010, a considerable number of medical schools have implemented curricular advances in this theme, starting from learning activities for longitudinal follow-up of patients from the perspective of Lifestyle Medicine, to a complete curriculum integration, with insertion of lifestyle competences to all existing curriculum fields. Final Remarks: The development of a Lifestyle Medicine curriculum is feasible, and early analysis of these strategies shows that students have improved confidence in prescribing and guiding lifestyle changes for patients with risk factors for noncommunicable chronic diseases.
\end{abstract}

Key words: Lifestyle, Medical Education, Curriculum.

\section{RESUMEN}

Objetivo: El objetivo de esta revisión fue identificar evidencia científica relacionada con el uso de estrategias para implementar un programa de Medicina de Estilo de Vida en escuelas de medicina. Revisión Bibliográfica: La Medicina de Estilo de Vida (MEV) es una especialidad médica que enfatiza el consejo médico sobre comportamientos que pueden mejorar la salud y la calidad de vida de los pacientes. Los pilares de esta metodología incluyen asesoramiento dietético, asesoramiento de actividad física, cambio de comportamiento, salud del sueño, dejar de fumar, consumo responsable de alcohol, bienestar emocional y

1 Universidade do Estado do Pará, Belém-PA;

2 Universidade Federal do Pará, Belém-PA *E-mail: santos-lobato@hotmail.com 
reducción del estrés. Tras la publicación de las competencias de MEV para la educación médica en 2010, un número considerable de escuelas de medicina han implementado avances curriculares en este tema, comenzando desde actividades de aprendizaje para el seguimiento longitudinal de pacientes desde la perspectiva de MEV para completar la integración curricular, incluyendo Habilidades de MEV para todos los ejes curriculares existentes. Consideraciones Finales: El desarrollo de un plan de estudios de MEV es factible y el análisis temprano de estas estrategias muestra que los estudiantes han mejorado la confianza en la prescripción y guía de cambios de estilo de vida para pacientes con factores de riesgo de enfermedades crónicas no transmisibles.

Palabras clave: Estilo de Vida, Educación Médica, Curriculum.

\section{INTRODUÇÃO}

A Medicina do Estilo de Vida (MEV) é uma disciplina clínica baseada em evidências, que enfatiza o aconselhamento médico sobre comportamentos que podem melhorar a saúde e qualidade de vida de pacientes (LIANOV L e JOHNSON M, 2010). Os pilares desta metodologia incluem aconselhamento alimentar, aconselhamento em atividade física, mudança de comportamento, saúde do sono, cessação do tabagismo, uso responsável do álcool, bem-estar emocional e redução do estresse (TRILK J, et al., 2019). Em 2018, a Associação Americana de Escolas Médicas reconheceu a MEV como uma das cinco especialidades médicas emergentes de maior destaque, devido a elevada importância na prevenção e manejo de doenças crônicas, e reforçou a necessidade de implementar este treinamento para futuros médicos, com o objetivo de reduzir os custos em saúde (TRILK JL, et al., 2019).

A prática da medicina preventiva visa primordialmente identificar e controlar os fatores de risco das doenças, em vez de direcionar o tratamento somente para as doenças em si (ANIL S, et al., 2016). Embora muitas organizações de saúde recomendem que os clínicos atuem na prevenção e promoção da saúde, baseando-se nas melhores evidências disponíveis, ainda há baixa adesão a essas recomendações (FRANK E, et al., 2013).

Infelizmente, os pacientes portadores das doenças crônicas de maior prevalência em nosso país (hipertensão arterial sistêmica e diabetes) possuem baixa adesão às mudanças de estilo de vida e ao autocuidado pessoal (SALIN AB, et al., 2019). Motivar pacientes para a mudança do comportamento em saúde é um verdadeiro desafio que depende de habilidades com o método. É importante enfatizar que o simples incentivo para mudanças de hábitos ao final de uma consulta produz resultados muito limitados. $O$ sucesso desta prática inclui principalmente planos de ações específicos em parceria com pacientes ou acompanhantes, de forma estruturada e longitudinal (LIANOV L e JOHNSON M, 2010).

Um dos motivos identificados para a falta de habilidades em MEV é a escassez na abordagem prática deste tema no contexto da educação médica (POLAK R, et al., 2014). Mesmo que seja responsabilidade do médico implementar a prevenção nos cuidados de saúde e direcionar seus pacientes para mudanças de estilo de vida, a maioria dos profissionais não possui conhecimentos, habilidades clínicas ou tempo disponível para esta orientação. De fato, uma elevada parcela dos profissionais de saúde não recebe treinamento adequado para fornecer aconselhamento sobre prevenção e manejo de doenças crônicas (TRILK J, et al., 2019).

Conforme enfatizado por Robiner W, et al. (2013), médicos são percebidos pela população como exemplos de autocuidado pessoal, além de serem considerados parte integrante do sistema de saúde e membros respeitados de suas comunidades. Eles servem, portanto, como um modelo e exercem influência no comportamento de seus pacientes, uma vez que suas ações pessoais complementam suas palavras e prescrições (ROBINER W, et al., 2013). Comportamentos pessoais pouco saudáveis e atitudes negativas em relação a estratégias preventivas podem levar a falta de credibilidade no atendimento médico, descontentamento pessoal no trabalho e resultados insatisfatórios de pacientes (FRANK E, et al., 2013).

Há evidências de que profissionais de saúde que tenham recebido formação em medicina preventiva, além de terem adquirido hábitos de vida mais saudáveis, também apresentem aperfeiçoamento nas atitudes em relação ao aconselhamento preventivo. No entanto, em países em desenvolvimento, a integração curricular 
direcionada para este tema ainda é escassa (ALBA LH, et al., 2015). Melhorar os resultados de saúde nos profissionais e na população inclui primordialmente o envolvimento da educação médica, uma vez que se deve ensinar aos alunos desde a faculdade sobre diretrizes eficientes, encorajando os futuros profissionais de saúde para o desempenho de um papel ainda mais ativo no acompanhamento longitudinal de seus pacientes (ROBINER W, et al., 2013).

Deste modo, o objetivo desta revisão foi identificar evidências científicas relacionadas ao uso de estratégias de implementação de um programa em MEV nas Escolas Médicas. Os estudos foram avaliados conforme a metodologia aplicada para determinar quais os meios utilizados para a abordagem do tema em educação médica, sendo então divididas em três grupos: propostas curriculares, estratégias de aprendizagem e atividades extracurriculares em MEV para estudantes de graduação.

\section{REVISÃO BIBLIOGRÁFICA}

\section{Propostas curriculares em medicina do estilo de vida}

Lianov L e Johnson M, (2010) publicaram no JAMA competências centrais em MEV para graduação médica, as quais incluem conhecimentos, habilidades de avaliação e habilidades de manejo. Como conhecimento, espera-se que os futuros médicos reconheçam as evidências de que mudanças comportamentais impactam positivamente em desfechos de saúde, e que saibam descrever como médicos podem engajar seus pacientes. Dentre as habilidades de avaliação, inclui-se a realização de anamnese e exame físico direcionados para os "sinais vitais" da MEV tais como uso de tabaco ou álcool, atividade física, nível de estresse, qualidade do sono e bem-estar emocional. Nas habilidades de manejo, espera-se que 0 médico desenvolva um plano de ações com prescrições de mudanças de estilo de vida para seus pacientes com fatores de risco para doenças crônicas.

A partir da publicação destas competências, um número considerável de escolas médicas tem implementado avanços curriculares em MEV. Os treinamentos dos estudantes incluem explicação sobre fisiologia, prevenção e tratamento de doenças crônicas, métodos de orientação de mudanças comportamentais e modelos de cuidados baseados em grupos com profissionais de educação física, enfermeiros, nutricionistas, psicólogos e coaches de bem-estar. Esta interação entre diferentes especialidades amplia a visão médica sobre métodos de abordagem do paciente, favorecendo a prática como parte de uma equipe interdisciplinar (TRILK J, et al., 2019).

Em 2013, o curso de medicina da Universidade da Carolina do Sul nos Estados Unidos e o Instituto de Medicina do Estilo de Vida da Universidade de Harvard promoveram um encontro, com o objetivo de introduzir a MEV no currículo da instituição. Os conteúdos de nutrição, exercício físico e mudanças comportamentais foram elencados como os mais importantes e não estavam representados dentro do projeto pedagógico do curso. As competências foram definidas ao longo de dois anos, com simpósios direcionados aos docentes do curso para que os mesmos compreendessem a importância do tema, investigando formas de integrar MEV nos eixos curriculares já existentes e antevendo possíveis barreiras (TRILK J, et al., 2016).

Em 2016, foi fundado o Conselho Colaborativo de Medicina do Estilo de Vida, o qual auxilia com a implementação curricular de escolas médicas dos Estados Unidos. Anualmente, as instituições médicas norteamericanas se reúnem neste Conselho para compartilharem desafios e casos de sucesso relacionados aos esforços na implementação curricular. Até 2018, um total de nove escolas americanas apresentaram suas propostas. Nas Universidades de Ohio e na de Toledo, o currículo foi iniciado a partir de uma disciplina eletiva com três temais centrais: atividade física, hábitos alimentares e manejo do estresse. Na Universidade de Virgínia, as atividades foram acrescentadas no estágio de Medicina de Família e Comunidade para estudantes do terceiro ano e direcionadas para o tratamento de pacientes com obesidade (MUSCATO D, et al., 2018).

$\mathrm{Na}$ Escola de Medicina Kaiser Permanente, nos Estados Unidos, foi implementado um currículo em espiral nos quais os conteúdos foram abordados em três fases. Inicialmente, os estudantes acessavam as evidências que ilustram a relação entre fatores de risco de estilo de vida e resultados de saúde. Em seguida, é destacada a relação entre os hábitos de saúde de um médico e os resultados de seus pacientes e, por fim, os estudantes 
desenvolvem o aprendizado de técnicas clínicas de intervenção em alimentação, atividade física, sono, uso de tabaco e álcool, manejo do estresse, bem-estar emocional e mudança de comportamento (MUSCATO D, et al., 2018).

Em 2019, foi desenvolvido um passo-a-passo demonstrando como ocorreu a elaboração do currículo em MEV da Universidade da Carolina do Sul. Nos primeiros semestres, os estudantes identificam os mecanismos bioquímicos pelos quais comportamentos não saudáveis podem levar ao desenvolvimento de doenças crônicas, de forma integrada ao eixo tutorial do curso (por exemplo, de que forma a atividade física influencia na fisiologia cardiovascular). Nos três anos seguintes, os alunos devem alcançar gradativamente um total de seis competências centrais, como aconselhar pacientes e familiares sobre as principais doenças crônicas relacionadas ao estilo de vida. O currículo é totalmente integrado aos demais eixos e possui uma carga horária total de 86 horas em MEV (TRILK J, et al., 2019).

A experiência da implementação da MEV em residências médicas pode ser utilizada como um exemplo de como estes temas podem ser adicionados também em rodízios práticos. Na Universidade da Califórnia, em San Diego, a MEV foi adicionada como módulo complementar ao programa de residência médica em medicina preventiva com ênfase principalmente para a atividade física. Foram acrescentados um total de seis módulos, os quais totalizaram doze semanas de treinamento. Modalidades integrativas como tai-chi, yoga, e mindfulness também foram incorporadas. Dentre os objetivos de aprendizagem, ao final do estágio o médico deveria ser capaz de descrever os princípios da prescrição de exercícios para indivíduos saudáveis e em risco de doenças crônicas, reconhecendo a indicação de cada tipo de atividade, além de estar apto a realizar o acompanhamento adequado destes pacientes (HILL, L, et al.,2015).

Outra estratégia de introdução da MEV em residência médica foi utilizada no Hospital Griffin, em Connecticut, para os programas de medicina preventiva e medicina interna. Neste projeto, a MEV foi adicionada como um módulo mais específico com duas semanas de duração. Concomitantemente, foram incluídas aulas didáticas e conferências sobre temas em MEV também nos demais rodízios relacionados aos programas. O treinamento prático enfatizou a aquisição de habilidades em entrevista motivacional e prescrição de exercício físico e a avaliação da aquisição de competências usou simulações de atendimento, com o uso do Exame Clínico Objetivo Estruturado (OSCE). De forma muito interessante, ao final do rodízio, os médicos relataram que houve melhora inclusive de seus próprios hábitos relacionados ao estilo de vida (NAWAZ H, et al., 2016).

O Colégio Americano de Medicina do Estilo de Vida destaca que o currículo em MEV deverá abordar as competências centrais a partir dos seguintes temas: nutrição, atividade física, redução do uso de álcool, saúde do sono, redução do estresse, cessação do tabagismo, além de reforçar treinamentos em terapia médica nutricional, introdução à medicina culinária e redução de fatores de risco cardiovascular. $O$ objetivo do programa é possibilitar a aquisição de práticas de liderança, de modo que o médico seja um provedor de comportamentos saudáveis para seus pacientes e demais profissionais atuantes no mesmo ambiente de trabalho (PERE D, 2017).

Deve-se enfatizar que, para uma correta incorporação curricular em MEV na educação médica, é fundamental que as instituições se apoiem em metas de implementação, as quais incluem avaliação estrutural e estabelecimento de parcerias com unidades básicas de saúde para uma maior aproximação da comunidade. Outra estratégia é o envolvimento de todo o corpo de docentes, uma vez que para uma completa integração, a iniciativa deverá emergir de um esforço conjunto desde o ciclo básico. Buscar o engajamento dos estudantes também é um ponto crítico para a aceitação e disseminação das práticas em MEV, portanto os mesmos também devem ter representação no planejamento das atividades a serem desempenhadas (PHILLIPS E, et al., 2015).

\section{Estratégias de aprendizagem em medicina do estilo de vida}

De forma alternativa, muitas Escolas Médicas implantaram novas estratégias de aprendizagem como forma de abordar a MEV na graduação, antes de uma reforma curricular completa. Em 2012, a Escola de Medicina de Harvard idealizou um programa de estágio de um ano em MEV. Para a elaboração do programa, 
os autores inicialmente acessaram o reconhecimento da importância de cinco domínios em medicina preventiva e realizaram um estudo piloto para a avaliação de competências previamente estabelecidas entre a equipe multiprofissional da própria universidade. Os domínios definidos na análise dos resultados incluíram nutrição, atividade física, manejo do estresse, mudanças comportamentais e saúde pessoal (POLAK R, et al., 2014).

Previamente às atividades em MEV, $30 \%$ dos estudantes de Universidade Harvard acreditavam que dizer ao paciente que sua obesidade provavelmente o mataria era uma abordagem válida para motivar um paciente a adotar um estilo de vida mais saudável. Ao final, houve melhora da confiança dos alunos em aconselhamento sobre mudanças de estilo de vida, exercícios, nutrição, sono, manejo do estresse e cessação do tabagismo, além de conscientização sobre a necessidade de aprimorar o aprendizado em abordagens motivacionais (FRATES E, et al., 2016).

$\mathrm{Na}$ Faculdade de Medicina de Chicago, foi implementado um treinamento intitulado "Mantenha seu coração saudável" para estudantes em estágios pré-clínicos, objetivando orientação sobre prevenção de doenças cardiovasculares. As atividades se dividiam entre aulas didáticas sobre epidemiologia e prevenção de doenças cardiovasculares e aconselhamento para pacientes simulados como treinamento de entrevista motivacional. Após as atividades iniciais, os estudantes realizavam atendimentos de pacientes da comunidade, sob supervisão de um coordenador do programa. Ao final do treinamento, $92 \%$ dos estudantes informaram melhora do conhecimento sobre prevenção de doenças cardiovasculares e $88 \%$ afirmaram que a prática suplementou o aprendizado em sala de aula, sugerindo que a atividade fosse adicionada ao programa curricular regular da instituição (SHAH N, et al., 2016).

Em escolas médicas de Israel, foi desenvolvida uma estratégia direcionada para práticas em MEV, onde inseriu-se atividades longitudinais, nas quais cada aluno do curso realiza o seguimento de um paciente em mudanças de estilo de vida pelo período de um ano. Esta atividade ocorre durante os quatro primeiros anos do curso, com aumento progressivo da complexidade do atendimento, de modo que ao final dos quatro anos, cada aluno terá acompanhado um total de quatro pacientes com doenças crônicas de forma longitudinal, sendo co-responsável pela evolução de saúde destes indivíduos. Mensalmente, os estudantes participam de sessões moderadas por professores do programa e especialistas em mudança de comportamento, onde apresentam a evolução de seu paciente, recebem feedback e discutem mais sobre a educação em medicina preventiva. $\mathrm{Na}$ avaliação curricular, os alunos informaram que as práticas foram fundamentais para a melhora da confiança na realização de prescrições e orientações em mudanças de estilo de vida (POLAK R, et al., 2017).

\section{Atividades extracurriculares em medicina do estilo de vida para estudantes de medicina}

Além de desenvolver competências relacionadas à orientação em saúde, as instituições de ensino superior também encorajam mudanças comportamentais dos próprios estudantes, direcionando carga horária extracurricular para programas de atividade física e exercícios, sessões de meditação mindfulness, experiências em medicina culinária e cultivo de hortas orgânicas (TRILK J, et al., 2019).

As análises precoces destas estratégias têm demonstrado resultados positivos, com aumento do engajamento dos futuros profissionais de saúde nesta prática, porém a adequação e adesão das escolas médicas a estas atividades ainda é incipiente (POLAK R, et al., 2015).

Tovar G, et al. (2016) comentam que se a educação médica obtiver êxito sobre a consciência do estudante ao passar por uma faculdade de Medicina, espera-se que todos eles ao final do curso sejam ativos. No entanto, o que foi observado no resultado do estudo dos mesmos autores, conduzido na Colômbia, é que a realidade dentro da faculdade se torna diferente, uma vez que a maior parte dos estudantes envolvidos na pesquisa eram sedentários e o índice de sobrepeso e obesidade maior com o passar dos semestres. Devido a isto, os autores sugeriram que os hábitos dos estudantes de Medicina também precisam ser avaliados continuamente e a adoção de práticas saudáveis deve fazer parte das competências adquiridas durante a formação profissional. Portanto, a universidade deve proporcionar condições adequadas para alcançar este 
objetivo, de modo que o estudante aprenda a partir de sua própria construção e vivência, resultando na formação de médicos que orientam melhor seus pacientes sobre o tema (TOVAR G, et al., 2016).

O uso de diários reflexivos se mostrou uma estratégia interessante para encorajar futuros médicos a melhorarem seus próprios hábitos em estilo de vida. Além disso, o ato de praticar o que se orienta auxilia na melhora da confiança quanto ao aconselhamento de pacientes, concomitantemente ao aprendizado sobre entrevista motivacional e prescrição de exercícios físicos (NAWAZ H, et al., 2016). A inclusão de um modelo de saúde para estudantes é essencial, pois além de basear-se no impacto positivo de ter um estudante de medicina mais saudável, também se traduz na melhora dos comportamentos de saúde de cada um de seus futuros pacientes (PHILLIPS E, et al., 2015).

A implementação da MEV deverá, portanto, percorrer um caminho que promova a integração completa da especialidade na educação médica. Inicialmente, deve-se estimular a adoção de hábitos de vida saudável pelos estudantes de medicina, com incentivo a atividades extracurriculares que contribuam com a carga horária complementar para a formação. Elaboração de estratégias de aprendizagem onde os estudantes tenham possibilidade de realizar o acompanhamento longitudinal de pacientes sob a ótica da MEV é uma abordagem interessante na etapa subsequente. O objetivo final deverá ser a integração curricular completa, de modo que a MEV esteja inserida em todos os eixos curriculares, durante os quatro primeiros anos do curso de medicina (Figura 1).

Figura 1 - Métodos de inserção da Medicina do Estilo de Vida (MEV) em Educação Médica.

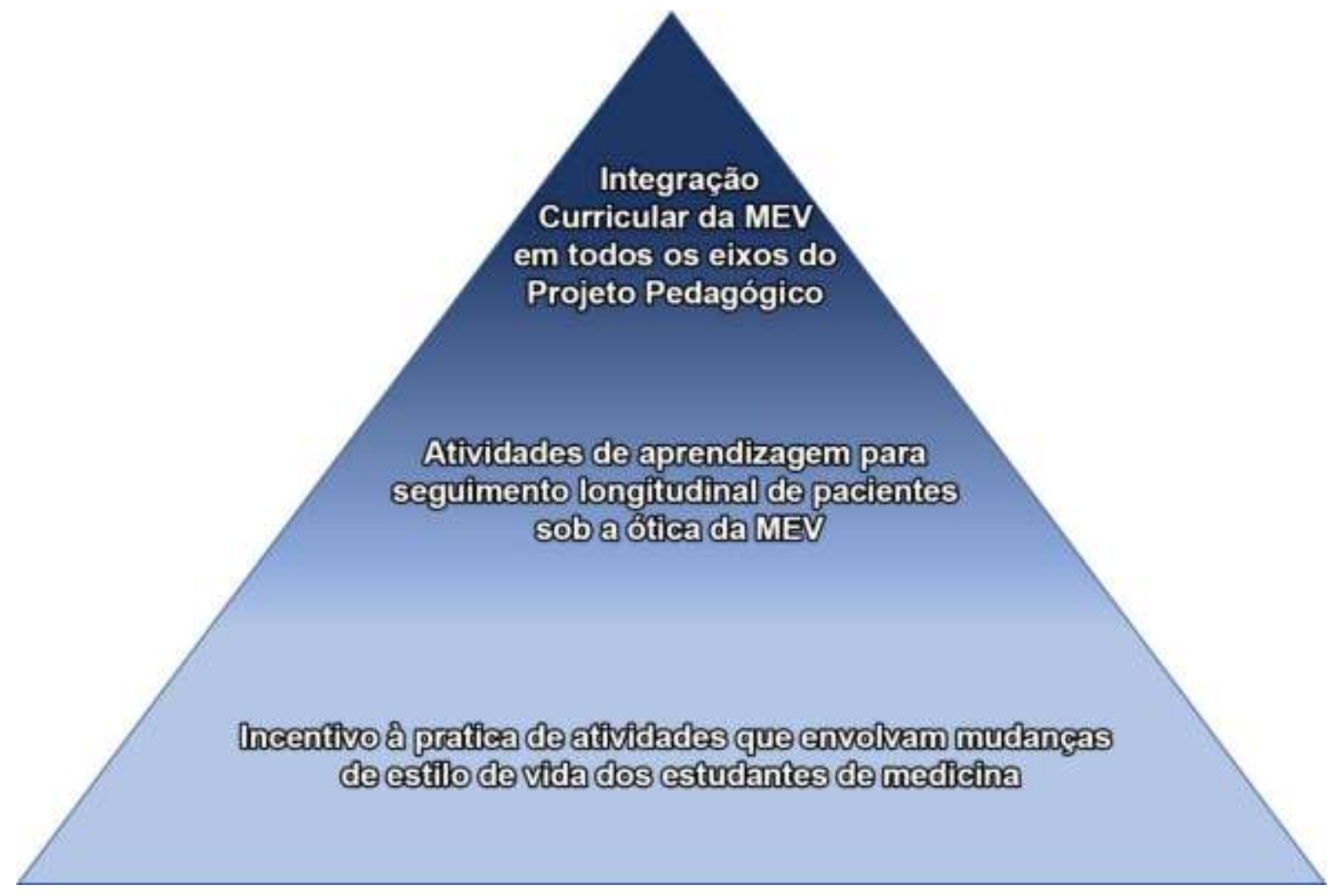

Fonte: Santos-Lobato EV et. al., 2019.

O desenvolvimento de um currículo em MEV para graduação médica é possível e viável, devendo emergir de um esforço contínuo, com treinamento, avaliações práticas e medidas de desempenho que auxiliem os estudantes a alcançarem as competências definidas no planejamento (LIANOV L e JOHNSON M, 2010). Se a missão e plano de implementações forem claros, iniciando a partir de pequenas etapas, é possível que o currículo em MEV seja desenvolvido de forma complementar e não competitivo ou desagregado ao ensino médico tradicional (TRILK JL, et al., 2019). 


\section{CONSIDERAÇÕES FINAIS}

A reforma curricular das escolas médicas deve incluir treinamento em MEV para auxiliar na formação de profissionais preparados para o crescente desafio de orientar prevenção e manejo de doenças crônicas, reduzindo a morbimortalidade e os custos em saúde para o tratamento destas complicações. A inserção da MEV em educação médica pode iniciar com a realização de atividades de aprendizagem independentes e com o incentivo à adoção de práticas de vida saudável pelos futuros profissionais de saúde, no entanto, é fundamental que progressivamente a especialidade esteja integrada aos demais eixos curriculares do curso em formato espiral. Para a inserção de competências nesta área, o plano de atividades deve ser claro, iniciando de forma gradativa e deve percorrer todos os semestres do curso. Com esta transformação da educação médica, os futuros profissionais terão ferramentas necessárias para atender a este novo sistema de assistência que objetiva abordar com sucesso os fatores de risco que refletem no desenvolvimento de doenças crônicas não transmissíveis.

\section{REFERÊNCIAS}

1. ALBA LH, et al. Attitude toward preventive counseling and healthy practices among medical students at a Colombian university, Frontiers of Medicine, 2015; 9(2): 251-259.

2. ANIL S, et al. Effectiveness of preventive medicine education and its determinants among medical students in Malaysia. Frontiers of Medicine, 2016; 10(1): 91-100.

3. FRANK E, et al. Do US medical students report more training on evidence-based prevention topics. Health Education Research, 2013; 28(2): 265-275.

4. FRATES E, et al. Increasing Knowledge and Confidence in Behavioral Change: a pilot study. The Primary Care Companion for CNS Disorders, 2016; 18(6).

5. HILL, L, et al. Training on exercise is medicine. Within an integrative medicine curriculum. American Journal of Preventive Medicine, 2015; 49(5S3): 278-284.

6. LIANOV L e JOHNSON M. Physician competencies for prescribing lifestyle medicine. JAMA, 2010; 304(2): 202-203.

7. MUSCATO D, et al. Lifestyle Medicine Education Collaborative (LMEd): "Champions of Change" Medical School Leaders Workshop. American Journal of Lifestyle Medicine, 2018; 12(5): 382-386.

8. NAWAZ $\mathrm{H}$, et al. Lifestyle Medicine curriculum for a preventive medicine residency program implementation and outcomes. Medical Education Online, 2016; 8(21): 29339.

9. PERE D. Building physician competency in Lifestyle Medicine: a model for health improvement. American Journal of Preventive Medicine, 2017; 52(2): 260-261.

10. PHILLIPS E, et al. Including lifestyle medicine in undergraduate medical curricula. Medical Education Online, 2015; 3(20): 26150.

11. POLAK R, et al. Bridging the gap - planning Lifestyle Medicine fellowship curricula: A cross sectional study. BMC Medical Education, 2014; 30(14):1-7.

12. POLAK R, et al. Lifestyle Medicine Education. American Journal of Lifestyle Medicine, 2015; 9(5):361-367.

13. POLAK R, et al. Medical students as health coaches: Implementation of a student-initiated Lifestyle Medicine curriculum. Israel Journal of Health Policy Research, 2017; 10(6).

14. ROBINER W, et al. Promoting health behaviors in medical education. Clinical Teacher, 2013; 10(3): 160-164.

15. SHAH N, et al. Development and evaluation of a service-learning model for preclinical student education in cardiovascular disease prevention. Advances in Medical Education and Practice, 2016; 11(7): 153-161.

16. TOVAR G, et al. Actividad física y consejería en estudiantes universitarios de primero y quinto año de medicina de Bogotá, Colombia. Universidad y Salud. 2016; 18(1): 16-23.

17. TRILK J, et al. Advancing Lifestyle Medicine Education in Undergraduate Medical School Curricula Through the Lifestyle Medicine Education Collaborative (LMEd). American Journal of Lifestyle Medicine. 2016; 12(5): 412-418.

18. TRILK J, et al. Design and Implementation of a Lifestyle Medicine Curriculum in Undergraduate Medical Education. American Journal of Lifestyle Medicine. 2019; 13(6): 574-585.

19. TRILK JL, et al. Including Lifestyle Medicine in Medical Education: Rational e for American College of Preventive Medicine/American Medical Association Resolution 959. American Journal of Preventive Medicine. 2019; 65(5): 169175. 